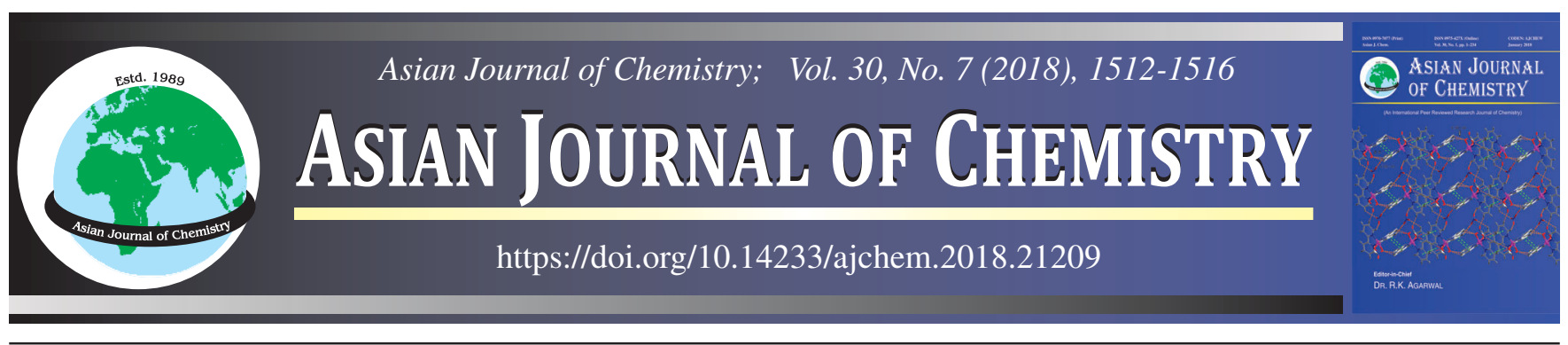

\title{
Chitosan-SO ${ }_{3} \mathrm{H}$ : A Green Approach to 2-Aryl/Heteroaryl Benzothiazoles under Solvent-Free Conditions at Room Temperature
}

\section{Surendra Bose Bathula ${ }^{1,2, *}$, Mukkanti KhagGa ${ }^{1}$ and Hariharakrishnan Venkatasubramanian ${ }^{3}$}

${ }^{1}$ Chemistry Division, Institute of Science and Technology, JNT University, Kukatpally, Hyderabad-500 072, India

${ }^{2}$ Alekhya Drugs Pvt. Ltd, Plot No: 145-150, IDA-Kondapalli, Ibrahimpatnam, Vijayawada-521 228, India

${ }^{3}$ RA Chem Pharma Ltd., Plot No. 26 \& 27, Technocrats Industrial Estate, Balanagar, Hyderabad-500 037, India

*Corresponding author: E-mail: surendrabose.bathulajntuh@gmail.com

Received: 1 December 2017; Accepted: 9 February 2018;

Published online: 31 May 2018;

AJC-18922

An efficient green protocol have been developed for the synthesis of 2-aryl/heteroaryl benzothiazole derivatives by intramolecular cyclocondensation of 2-mercaptoaniline with various substituted aryl/heteroaryl aldehydes using chitosan- $\mathrm{SO}_{3} \mathrm{H}$ as an efficient biocompatible and reusable heterogenous solid acid catalyst in presence of air under solvent free conditions at room temperature. ${ }^{1} \mathrm{H}$ NMR and ${ }^{13} \mathrm{C} \mathrm{NMR}$ spectra recorded are in agreement with the reported data.

Keywords: Green synthesis, Chitosan-SO $3 \mathrm{H}$, Benzothiazoles, Intramolecular cyclocondensation, Solvent-free condition.

\section{INTRODUCTION}

Benzothiazole ring is a fused bicyclic ring often constructed from acyclic reactants [1]. This core nucleus bearing $\mathrm{N}$ and $\mathrm{S}$ atoms at symmetrical position have been studied extensively owing to their interesting pharmacological activities. Especially, among those 2-arylbenzothiazoles (Fig. 1) are of great interest as these structural frameworks have proved to be as an important class of biological active motifs and hence play a pivotal role in the field of medicinal and industrial chemistry. They are the basic constituents of several antitumor, anticonvulsant, antiviral and anticancer drugs [2].

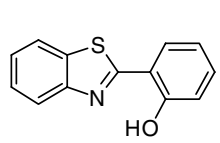

Replication \& Mitosis Inhibitors

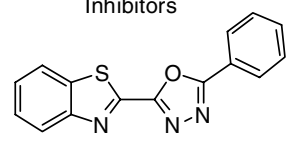

Antibacterial agent

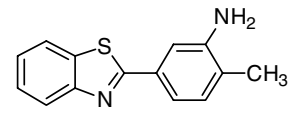

Topoisomerase II inhibitors

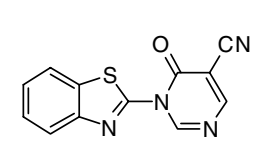

Antimicrobial agent

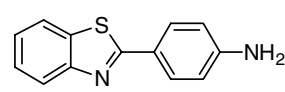

Anticancer agent

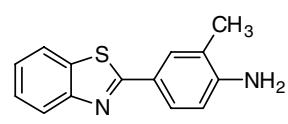

Antitumor reagent
Fig: 1. Biological activity profile of certain 2-substituted benzothiazole scaffolds [Ref. 3]

Traditionally, the common synthetic strategies to construct these bioactive compounds relies on majorly condensation of 2 -mercaptoaniline with aldehydes, $\beta$-diketones, carboxylic acids, acid chlorides or esters via Hofmann synthesis [4], oxidative intramolecular cyclization of thiobenzanilides via Jacobson cyclization [5] and palladium-catalyzed coupling of 2-bromobenzothiazole with arylboronic acids via Suzuki biaryl coupling [6].

However, many of the reported methods are quite effective and useful, but suffer by using acidic reagents or hazardous organic solvents that are not environmentally compatible. Moreover, harsh reaction conditions, multistep process, longer reaction times, higher temperatures, use of expensive reagents, metal oxidants and produce a large amounts of waste with observed side product formation are the other drawbacks of these methodologies. Consequently, there is a need to overcome the above limitations by developing an efficient, simple and green methodology for the synthesis of 2-substituted benzothiazoles in terms of selectivity, reusability and biocompatibility.

Recently, the direction of science and technology has been shifting more towards eco-friendly, natural product resources and reusable catalysts. Thus natural biopolymers have emerged as potential candidates for preparing solid acid support catalysts enabling high selectivity of the reactions under solventfree conditions [7]. Researchers have demonstrated that solventfree organic syntheses are generally faster, selective, higher yielding with cleaner products, environmentally benign and involve simple operational procedure as compared to the classical reactions [8]. To the best of our knowledge, as far as 
concerned to the literature revealed there are no reports found using this present methodology. We wish to report herein a simple procedure for the synthesis of 2-substituted benzothiazoles using chitosan- $\mathrm{SO}_{3} \mathrm{H}$ as a reusable solid acid catalyst under solvent-free conditions at room temperature with high selectivity.

Chitosan is a linear polyamine derivative briefed as [poly( $\beta$-1/4)-2-amino-2-deoxy-D-glucopyranose] obtained by partial and controlled deacetylation of chitin [9]. Chitosan can easily be subjected to a variety of chemical modifications. One such chemical modification is sulfonation at the reactive amine function to produce chitosan- $\mathrm{SO}_{3} \mathrm{H}\left(\mathrm{CS}-\mathrm{SO}_{3} \mathrm{H}\right)$ and has been used as a solid acid catalyst in many organic reactions [10]. Prompted by these reports, we herein report $\mathrm{CS}-\mathrm{SO}_{3} \mathrm{H}$ as an efficient, reusable heterogeneous solid acid catalyst for the synthesis of 2-aryl/heteroaryl benzothaizole derivatives under solvent free conditions at room temperature.

\section{EXPERIMENTAL}

${ }^{1} \mathrm{H}$ NMR and ${ }^{13} \mathrm{C}$ NMR spectra were recorded on Bruker$\mathrm{AV}$ (400 and $100 \mathrm{MHz}$, respectively) spectrometer by using $\mathrm{CDCl}_{3}$ solvent and tetramethylsilane (TMS) as an internal standard. The FT-IR spectra were obtained with $\mathrm{KBr}$ pellets in the range $4000-400 \mathrm{~cm}^{-1}$ with a Perkin Elmer 550 spectrometer. Melting points were uncorrected and determined in open capillary tubes using sulphuric acid bath and Mass spectra on an Agilent LC-MS instrument giving only $\mathrm{M}^{+}$values in $\mathrm{Q}+1$ mode. All starting materials, chitosan were purchased from TCI and Alfa-aesar.

General procedure for the preparation of 2-aryl/heteroaryl benzothiazoles catalyzed by chitosan- $\mathrm{SO}_{3} \mathrm{H}$ : In a round bottom equipped flask 2-mercaptoaniline (1) (1 mmol), substituted aryl/heteroaryl aldehydes $\mathbf{2}(\mathbf{a}-\mathbf{o})$ (1 $\mathrm{mmol}$ ) and CS$\mathrm{SO}_{3} \mathrm{H}(2 \mathrm{~mol} \%)$ is stirred at room temperature for $30 \mathrm{~min}$ in presence of oxygen balloon. The reaction was monitored by using TLC. Upon completion of the reaction, the reaction mass was quenched to absolute ethanol. The precipitated $\mathrm{CS}-\mathrm{SO}_{3} \mathrm{H}$ was filtered and reused for subsequent reactions. The filterate was concentrated and obtained the curde product. Further the crude product was recrystallized from ethanol and obtained pure products $\mathbf{3}(\mathbf{a}-\mathbf{0})$.

\section{Physical and spectral data}

2-Phenylbenzothiazole (3a): White solid, m.p.: 111-112 ${ }^{\circ} \mathrm{C}$ (Lit. [11] 110-111 ${ }^{\circ} \mathrm{C}$ ); IR (KBr, $\left.v_{\max }, \mathrm{cm}^{-1}\right)$ : 3066, 3017, 2835, 1608, 1587, 1476,1430, 830, 763; ${ }^{1} \mathrm{H}$ NMR (400 MHz, $\left.\mathrm{CDCl}_{3}, \mathrm{ppm}\right) \delta 7.37(\mathrm{t}, J=8.0 \mathrm{~Hz}, 1 \mathrm{H}), 7.39-7.51(\mathrm{~m}, 4 \mathrm{H})$, $7.89(\mathrm{~d}, J=8.0 \mathrm{~Hz}, 1 \mathrm{H}), 8.06-8.10(\mathrm{~m}, 3 \mathrm{H}) ;{ }^{13} \mathrm{C}$ NMR $(100$ $\mathrm{MHz}, \mathrm{CDCl}_{3}$, ppm) $\delta 121.59,123.24,125.16,126.29,127.56$, $128.99,130.93,133.64,135.07,154.16,168.03 ; \mathrm{MS}(\mathrm{m} / z)$ $[\mathrm{M}+\mathrm{H}]^{+}: 212$.

2-(4-Methylphenyl)benzothiazole (3b): White solid; m.p.: 82-84 ${ }^{\circ} \mathrm{C}$ (Lit. [12] 83-85 ${ }^{\circ} \mathrm{C}$ ); IR $\left(\mathrm{KBr}, v_{\max }, \mathrm{cm}^{-1}\right)$ : 3024, 2905, 1610, 1519, 1480, 1435, 1383, 1312, 821, 759; ${ }^{1} \mathrm{H}$ NMR (400 MHz, $\left.\mathrm{CDCl}_{3}, \mathrm{ppm}\right) \delta 8.06(\mathrm{~d}, J=8.0 \mathrm{~Hz}, 1 \mathrm{H}), 7.98(\mathrm{~d}, J$ $=8.0 \mathrm{~Hz}, 2 \mathrm{H}), 7.89(\mathrm{~d}, J=8.0 \mathrm{~Hz}, 1 \mathrm{H}), 7.48(\mathrm{t}, J=7.6 \mathrm{~Hz}$, $1 \mathrm{H}), 7.37$ (t, $J=7.4 \mathrm{~Hz}, 1 \mathrm{H}), 7.30(\mathrm{~d}, J=8.0 \mathrm{~Hz}, 2 \mathrm{H}), 2.42$ (s, $3 \mathrm{H}) ;{ }^{13} \mathrm{C} \mathrm{NMR}\left(100 \mathrm{MHz}, \mathrm{CDCl}_{3}, \mathrm{ppm}\right) \delta 168.2,154.2,141.3$,
135.0, 131.1, 129.7, 127.5, 126.2, 125.0, 123.1, 121.5, 21.4; $\operatorname{MS}(\mathrm{m} / \mathrm{z})[\mathrm{M}+\mathrm{H}]^{+}: 226$.

2-(4-Methoxyphenyl)benzothiazole (3c): White solid; m.p.: $120-122{ }^{\circ} \mathrm{C}$ (Lit. [13] 120-122 ${ }^{\circ} \mathrm{C}$ ); IR ( $\left.\mathrm{KBr}, v_{\max }, \mathrm{cm}^{-1}\right)$ : 3021, 3048, 2837, 1609, 1590, 1483, 830; ${ }^{1} \mathrm{H}$ NMR (400 MHz, $\left.\mathrm{CDCl}_{3}, \mathrm{ppm}\right) \delta 8.04(\mathrm{~d}, J=8.4 \mathrm{~Hz}, 3 \mathrm{H}), 7.88(\mathrm{~d}, J=8.0 \mathrm{~Hz}$, $1 \mathrm{H}), 7.47$ (t, $J=7.6 \mathrm{~Hz}, 1 \mathrm{H}), 7.35$ (t, $J=7.4 \mathrm{~Hz}, 1 \mathrm{H}), 7.00$ (d, $J=8.4 \mathrm{~Hz}, 2 \mathrm{H}), 3.88(\mathrm{~s}, 3 \mathrm{H}) ;{ }^{13} \mathrm{C} \mathrm{NMR}\left(100 \mathrm{MHz}, \mathrm{CDCl}_{3}\right.$, ppm) $\delta 167.8,162.0,154.3,134.9,129.1,126.5,126.1,124.7$, 122.8, 121.4, 114.4, 55.4; MS (m/z) $[\mathrm{M}+\mathrm{H}]^{+}: 242$.

2-(2-Hydroxyphenyl)benzothiazole (3d): Yellow solid; m.p.: $127-128^{\circ} \mathrm{C}$ (Lit. [14] 126-128 ${ }^{\circ} \mathrm{C}$ ); IR (KBr, $\left.v_{\max }, \mathrm{cm}^{-1}\right)$ : 3285, 3090, 2900, 1619, 1590, 1490, 1423, 874, 751; ${ }^{1} \mathrm{H}$ NMR (400 MHz, $\left.\mathrm{CDCl}_{3}, \mathrm{ppm}\right) \delta 6.95-699(\mathrm{t}, 1 \mathrm{H}, J=8.0 \mathrm{~Hz}, \mathrm{Ar}-\mathrm{H})$ 7.12 (d, 1H, $J=8.0 \mathrm{~Hz}, \mathrm{Ar}-\mathrm{H}), 7.38-7.44$ (m,2H, Ar-H) 7.50$7.54(\mathrm{t}, 1 \mathrm{H}, J=8.4 \mathrm{~Hz}, \mathrm{Ar}-\mathrm{H}) 7.71(\mathrm{~d}, 1 \mathrm{H}, J=8.0 \mathrm{~Hz}, \mathrm{Ar}-\mathrm{H})$ $7.92(\mathrm{~d}, 1 \mathrm{H}, J=8.4 \mathrm{~Hz}, \mathrm{Ar}-\mathrm{H}) 8.01(\mathrm{~d}, 1 \mathrm{H}, J=8.0 \mathrm{~Hz}, \mathrm{Ar}-\mathrm{H})$ 12.54 (s, $1 \mathrm{H}, \mathrm{OH}) ;{ }^{13} \mathrm{C} \mathrm{NMR}\left(100 \mathrm{MHz}, \mathrm{CDCl}_{3}, \mathrm{ppm}\right) \delta 116.8$, 117.9, 119.5, 121.5, 122.2, 125.6, 126.7, 128.4, 132.6, 132.8, 151.8, 157.9, 169.4; MS $(\mathrm{m} / \mathrm{z})[\mathrm{M}+\mathrm{H}]^{+}: 228$.

2-(4-Bromophenyl)benzothiazole (3e): White solid; m.p.: 129-131 ${ }^{\circ} \mathrm{C}$ (Lit. [15] 130-132 ${ }^{\circ} \mathrm{C}$ ); IR ( KBr, $\left.v_{\max }, \mathrm{cm}^{-1}\right)$ : 1503 , 1392, 1311, 1224, 968, 820, 754, 722, 477; ${ }^{1} \mathrm{H}$ NMR (400 $\left.\mathrm{MHz}, \mathrm{CDCl}_{3}, \mathrm{ppm}\right) \delta=8.06(\mathrm{~d}, J=8.2 \mathrm{~Hz}, 1 \mathrm{H}), 7.95(\mathrm{~d}, J=$ $8.6 \mathrm{~Hz}, 2 \mathrm{H}), 7.89$ (d, J = 7.8 Hz, 1H), 7.64- 7.60 (m, 2H), 7.50 (t, $J=7.4 \mathrm{~Hz}, 1 \mathrm{H}), 7.39$ (t, $J=7.6 \mathrm{~Hz}, 1 \mathrm{H}) ;{ }^{13} \mathrm{C} \mathrm{NMR}(100 \mathrm{MHz}$, $\left.\mathrm{CDCl}_{3}, \mathrm{ppm}\right) \delta=166.7,154.1,135.0,132.5,132.2,128.9$, 126.5, 125.4, 125.4, 123.3, 121.6; MS (m/z) $[\mathrm{M}+\mathrm{H}]^{+}: 289$.

2-(4-Chlorophenyl)benzothiazole (3f): White solid; m.p.: 114- $116^{\circ} \mathrm{C}$ (Lit. [16] 116-118 ${ }^{\circ} \mathrm{C}$ ); IR ( KBr, $\left.\nu_{\max }, \mathrm{cm}^{-1}\right)$ : 3055 , $2358,1560,1455,1430,1317,1275,1060,965,750,725 ;{ }^{1} \mathrm{H}$ NMR (400 MHz, $\left.\mathrm{CDCl}_{3}, \mathrm{ppm}\right) \delta$ 7.37-7.39 (m, 2H, Ar-H) 7.51-7.53 (d, 2H, $J=7.6 \mathrm{~Hz}, \mathrm{Ar}-\mathrm{H})$ 7.58-7.61 (m, 1H, Ar-H) 7.77-7.79 (m, 1H, Ar-H) 8.19-8.22 (d, 2H, J=7.6 Hz, Ar-H); ${ }^{13} \mathrm{C} \mathrm{NMR}\left(100 \mathrm{MHz}, \mathrm{CDCl}_{3}, \mathrm{ppm}\right) \delta 121.8,123.1,125.2$, 126.8, 129.1, 129.8, 132.7, 135.2, 137.1,154.4, 166.2; MS $(\mathrm{m} / \mathrm{z})[\mathrm{M}+\mathrm{H}]^{+}: 247$.

2-(4-Fluorophenyl)benzothiazole (3g): White solid; m.p.: 97-98 ${ }^{\circ} \mathrm{C}$ (Lit. [15] 98-100 ${ }^{\circ} \mathrm{C}$ ); IR (KBr, $v_{\max }, \mathrm{cm}^{-1}$ ): 1710, 1520, 1362, 1226, 1092,967, 837, 756, 728, 499; ${ }^{1} \mathrm{H}$ NMR (400 MHz, $\left.\mathrm{CDCl}_{3}, \mathrm{ppm}\right) \delta=8.09-8.04$ (m, 3H), 7.88 $(\mathrm{d}, J=7.9 \mathrm{~Hz}, 1 \mathrm{H}), 7.49(\mathrm{t}, J=7.8 \mathrm{~Hz}, 1 \mathrm{H}), 7.38(\mathrm{t}, J=7.6$ $\mathrm{Hz}, 1 \mathrm{H}), 7.20-7.14(\mathrm{~m}, 2 \mathrm{H}) ;{ }^{13} \mathrm{C} \mathrm{NMR}\left(100 \mathrm{MHz}, \mathrm{CDCl}_{3}, \mathrm{ppm}\right)$ $\delta=166.2(\mathrm{~d}, J=101.9 \mathrm{~Hz}), 163.2,154.1,135.0,130.0(\mathrm{~d}, J=$ $3.1 \mathrm{~Hz}), 129.5$ (d, $J=8.6 \mathrm{~Hz}), 126.4,125.2,123.2,121.6$, $116.1(\mathrm{~d}, J=22.0 \mathrm{~Hz})$; $\mathrm{MS}(\mathrm{m} / \mathrm{z})[\mathrm{M}+\mathrm{H}]^{+}: 230$.

2-(4-Trifluoromethylphenyl)benzothiazole (3h): White solid; m.p.: 160-162 ${ }^{\circ} \mathrm{C}$ (Lit. [17] 160-162 ${ }^{\circ} \mathrm{C}$ ); IR (KBr, $\nu_{\max }$, $\left.\mathrm{cm}^{-1}\right): 1483,1434,1323,1110,970,839,760,620,439 ;{ }^{1} \mathrm{H}$ NMR (400 MHz, $\left.\mathrm{CDCl}_{3}, \mathrm{ppm}\right) \delta 8.21(\mathrm{~d}, J=8.3 \mathrm{~Hz}, 2 \mathrm{H})$, $8.11(\mathrm{~d}, J=8.1 \mathrm{~Hz}, 1 \mathrm{H}), 7.93(\mathrm{~d}, J=8.0 \mathrm{~Hz}, 1 \mathrm{H}), 7.75(\mathrm{~d}, J=$ $8.3 \mathrm{~Hz}, 2 \mathrm{H}), 7.53$ (t, $J=7.4 \mathrm{~Hz}, 1 \mathrm{H}), 7.43$ (t, $J=7.5 \mathrm{~Hz}, 1 \mathrm{H})$; ${ }^{13} \mathrm{C} \mathrm{NMR}\left(100 \mathrm{MHz}, \mathrm{CDCl}_{3}, \mathrm{ppm}\right) \delta 166.0,154.0,136.8$, $135.2,132.3(\mathrm{q}, J=32.6 \mathrm{~Hz}), 127.8,126.7,126.0(\mathrm{q}, J=3.7$ $\mathrm{Hz}), 123.7$ (q, $J=270.8 \mathrm{~Hz}), 123.6,121.7$; MS $(\mathrm{m} / \mathrm{z})[\mathrm{M}+\mathrm{H}]^{+}$: 280 .

2-(3-Nitrophenyl)benzothiazole (3i): White solid; m.p.: 181-183 ${ }^{\circ} \mathrm{C}$ (Lit. [18] 181-182 ${ }^{\circ} \mathrm{C}$ ); IR ( $\left.\mathrm{KBr}, v_{\max }, \mathrm{cm}^{-1}\right)$ : 3039 , 
2936, 1522, 1460, 1345, 1103, 1041, 851, 750; ${ }^{1} \mathrm{H}$ NMR (400 $\left.\mathrm{MHz}, \mathrm{CDCl}_{3}, \mathrm{ppm}\right) \delta 8.94(\mathrm{~s}, 1 \mathrm{H}), 8.43(\mathrm{~d}, J=7.6 \mathrm{~Hz}, 1 \mathrm{H})$, $8.34(\mathrm{~d}, J=7.6 \mathrm{~Hz}, 1 \mathrm{H}), 8.13(\mathrm{~d}, J=8.0 \mathrm{~Hz}, 1 \mathrm{H}), 7.96(\mathrm{~d}, J=$ $8.0 \mathrm{~Hz}, 1 \mathrm{H}), 7.70(\mathrm{t}, J=7.6 \mathrm{~Hz}, 1 \mathrm{H}), 7.56(\mathrm{t}, J=7.6 \mathrm{~Hz}, 1 \mathrm{H})$, $7.46(\mathrm{t}, J=7.6 \mathrm{~Hz}, 1 \mathrm{H}) ;{ }^{13} \mathrm{C} \mathrm{NMR}\left(100 \mathrm{MHz}, \mathrm{CDCl}_{3}, \mathrm{ppm}\right) \delta$ 164.9, 153.9, 148.7, 135.3, 135.2, 133.0, 130.1, 126.8, 126.0, 125.1, 123.7, 122.3, 121.8; MS (m/z) $[\mathrm{M}+\mathrm{H}]^{+}: 257$.

2-(3-Bromo-4-methylphenyl)benzothiazole (3j): White solid, m.p.: $115-117^{\circ} \mathrm{C}$. (Lit. [19] $115-117{ }^{\circ} \mathrm{C}$ ); IR (KBr, $\left.v_{\max }, \mathrm{cm}^{-1}\right): 1472,1433,1375,1310,1284,973,824,756,724$, 433; ${ }^{1} \mathrm{H}$ NMR (400 MHz, $\left.\mathrm{CDCl}_{3}, \mathrm{ppm}\right) \delta 8.29(\mathrm{~d}, J=1.4 \mathrm{~Hz}$, $1 \mathrm{H}), 8.07(\mathrm{~d}, J=8.2 \mathrm{~Hz}, 1 \mathrm{H}), 7.90(\mathrm{~d}, J=7.9 \mathrm{~Hz}, 2 \mathrm{H}), 7.50(\mathrm{t}$, $J=7.7 \mathrm{~Hz}, 1 \mathrm{H}), 7.39(\mathrm{t}, J=7.3 \mathrm{~Hz}, 1 \mathrm{H}), 7.34(\mathrm{~d}, J=7.9 \mathrm{~Hz}$, $1 \mathrm{H}), 2.47$ (s, 3H); ${ }^{13} \mathrm{C}$ NMR $\left(100 \mathrm{MHz}, \mathrm{CDCl}_{3}, \mathrm{ppm}\right): \delta 166.3$, 154.0, 141.0, 135.0, 132.9, 131.2, 131.0, 126.4, 126.3, 125.5, 125.3, 123.2, 121.6; MS (m/z) $[\mathrm{M}+\mathrm{H}]^{+}: 303$.

2-(Naphthalen-2-yl)benzothiazole (3k): White solid; m.p.: $125-127^{\circ} \mathrm{C}$ (Lit. [20] $126-128^{\circ} \mathrm{C}$ ); IR (KBr, $v_{\max }, \mathrm{cm}^{-1}$ ): 1496, 1453, 1361, 1311, 981, 934, 852, 829, 747, 728, 476; ${ }^{1} \mathrm{H}$ NMR $\left(400 \mathrm{MHz}, \mathrm{CDCl}_{3}, \mathrm{ppm}\right) \delta 8.56(\mathrm{~d}, J=1.2 \mathrm{~Hz}, 1 \mathrm{H})$, $8.20(\mathrm{dd}, J=8.7 \mathrm{~Hz}, 1 \mathrm{H}), 8.11(\mathrm{~d}, J=8.2 \mathrm{~Hz}, 1 \mathrm{H}), 7.98-7.86$ (m, 4H), 7.56-7.49 (m, 3H), $7.40\left(\mathrm{t}, J=7.6 \mathrm{~Hz}, 1 \mathrm{H} ;{ }^{13} \mathrm{C}\right.$ NMR $\left(100 \mathrm{MHz}, \mathrm{CDCl}_{3}, \mathrm{ppm}\right) \delta 168.1,154.2,135.1,134.6,133.2$, 131.0; MS $(\mathrm{m} / \mathrm{z})[\mathrm{M}+\mathrm{H}]^{+}: 262$.

2-(Thiophen-2-yl)benzothiazole (3l): White solid; m.p.: 92-94 ${ }^{\circ} \mathrm{C}$ (Lit. [21] $\left.93-95^{\circ} \mathrm{C}\right)$; IR ( $\left.\mathrm{KBr}, v_{\max }, \mathrm{cm}^{-1}\right)$ : 3096, 3056, 1542, 1476, 1312, 1222, 912, 852, 826, 762, 714; ${ }^{1} \mathrm{H}$ NMR $\left(400 \mathrm{MHz}, \mathrm{CDCl}_{3}, \mathrm{ppm}\right) \delta 8.03(\mathrm{~d}, J=8.4 \mathrm{~Hz}, 1 \mathrm{H}), 7.85(\mathrm{~d}, J$ $=8.0 \mathrm{~Hz}, 1 \mathrm{H}), 7.66(\mathrm{~d}, J=3.2 \mathrm{~Hz}, 1 \mathrm{H}), 7.51-7.45(\mathrm{~m}, 2 \mathrm{H})$, $7.37(\mathrm{t}, J=7.6 \mathrm{~Hz}, 1 \mathrm{H}), 7.14(\mathrm{t}, J=4.2 \mathrm{~Hz}, 1 \mathrm{H}) ;{ }^{13} \mathrm{C} \mathrm{NMR}$ $\left(100 \mathrm{MHz}, \mathrm{CDCl}_{3}, \mathrm{ppm}\right) \delta 161.3,153.7,137.4,134.7,129.2$, $128.5,128.0,126.4,125.2,123.0,121.4 ; \mathrm{MS}(\mathrm{m} / \mathrm{z})[\mathrm{M}+\mathrm{H}]^{+}$: 218.

2-(Pyridin-3-yl)benzothiazole (3m): White solid; m.p.: $135-137{ }^{\circ} \mathrm{C}$ (Lit. [22] $\left.137-138^{\circ} \mathrm{C}\right)$; IR ( $\left.\mathrm{KBr}, v_{\max }, \mathrm{cm}^{-1}\right)$ : 3050, $3032,1586,1574,1426,1310,1234,964,814,766,702 ;{ }^{1} \mathrm{H}$ NMR (400 MHz, $\left.\mathrm{CDCl}_{3}, \mathrm{ppm}\right) \delta 9.28(\mathrm{~d}, J=1.6 \mathrm{~Hz}, 1 \mathrm{H})$, $8.70(\mathrm{dd}, J=0.8,4.4 \mathrm{~Hz}, 1 \mathrm{H}), 8.37-8.34(\mathrm{~m}, 1 \mathrm{H}), 8.09(\mathrm{~d}, J=$ $8.4 \mathrm{~Hz}, 1 \mathrm{H}), 7.91(\mathrm{~d}, J=8.0 \mathrm{~Hz}), 7.53-7.49(\mathrm{~m}, 1 \mathrm{H}), 7.43-$ 7.39 (m, 2H); ${ }^{13} \mathrm{C}$ NMR (100 MHz, $\mathrm{CDCl}_{3}$, ppm) $\delta 164.5$, 153.9, 151.5, 148.5, 134.9, 134.5, 129.6, 126.6, 125.6, 123.7, 123.4, 121.7; MS $(\mathrm{m} / \mathrm{z})[\mathrm{M}+\mathrm{H}]^{+}: 213$.

2-(Pyridin-4-yl)benzothiazole (3n): White solid; m.p.: 131-133 ${ }^{\circ} \mathrm{C}$ (Lit. [23] $\left.132-134{ }^{\circ} \mathrm{C}\right)$; IR ( $\left.\mathrm{KBr}, v_{\max }, \mathrm{cm}^{-1}\right)$ : 1433 , 979, 758, 728, 720; ${ }^{1} \mathrm{H}$ NMR (400 MHz, $\left.\mathrm{CDCl}_{3}, \mathrm{ppm}\right): \delta 8.78$ $(\mathrm{d}, J=4.8 \mathrm{~Hz}, 2 \mathrm{H}), 8.14(\mathrm{~d}, J=8.0 \mathrm{~Hz}, 1 \mathrm{H}), 7.96-7.95(\mathrm{~m}$, $3 \mathrm{H}), 7.56(\mathrm{t}, J=7.6 \mathrm{~Hz}, 1 \mathrm{H}), 7.47(\mathrm{t}, J=7.4 \mathrm{~Hz}, 1 \mathrm{H}) ;{ }^{13} \mathrm{C} \mathrm{NMR}$ $\left(100 \mathrm{MHz}, \mathrm{CDCl}_{3}, \mathrm{ppm}\right): \delta 165.1,154.0,150.7,140.5,135.2$, 126.8, 126.2, 123.9, 121.8, 121.2; MS $(\mathrm{m} / \mathrm{z})[\mathrm{M}+\mathrm{H}]^{+}: 213$.

2-(Furan-2-yl)benzothiazole (3o): Yellow solid; m.p.: $100-102{ }^{\circ} \mathrm{C}$ (Lit. [22] $102-104{ }^{\circ} \mathrm{C}$ ); IR (KBr, $v_{\max }, \mathrm{cm}^{-1}$ ): 3144 , $3122,3050,1598,1578,1434,1246,1114,898,748,730 ;{ }^{1} \mathrm{H}$ NMR (400 MHz, $\mathrm{CDCl}_{3}$, ppm) $\delta 6.57(\mathrm{dd}, J=1.5,7.4 \mathrm{~Hz}$, $1 \mathrm{H}), 7.17(\mathrm{~d}, J=6.9 \mathrm{~Hz}, 1 \mathrm{H}), 7.35$ (dt, $J=1.0,8.3 \mathrm{~Hz}, 1 \mathrm{H})$, $7.47(\mathrm{dt}, J=1.0,8.3 \mathrm{~Hz}, 1 \mathrm{H}), 7.55-7.59(\mathrm{~m}, 1 \mathrm{H}), 7.86(\mathrm{~d}, J=$ $7.3 \mathrm{~Hz}, 1 \mathrm{H}), 8.04(\mathrm{~d}, J=8.3 \mathrm{~Hz}, 1 \mathrm{H}) ;{ }^{13} \mathrm{C}$ NMR $(100 \mathrm{MHz}$, $\left.\mathrm{CDCl}_{3}, \mathrm{ppm}\right) \delta 111.3,112.4,121.4,123.0,125.1,126.4,134.1$, 144.6, 148.6, 153.6, 157.4; MS (m/z) $[\mathrm{M}+\mathrm{H}]^{+}: 202$.

\section{RESULTS AND DISCUSSION}

In continuation of our research on synthetic applications of green catalysts [24] for pharmacologically active heterocyclic compounds herein we wish to report a simple synthesis of 2-aryl/heteroaryl benzothiazoles by condensation of 2-mercaptoaniline with various substituted aryl/heteroaryl aldehydes under solvent-free conditions catalyzed by $\mathrm{CS}-\mathrm{SO}_{3} \mathrm{H}$ at room temperature as depicted in Scheme-I.

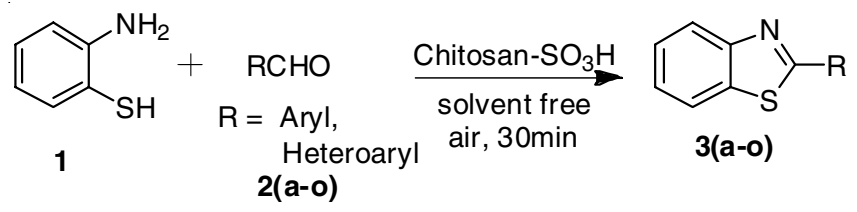

Scheme-I: Synthesis of 2-aryl/heteroaryl benzothiaozles

Initially, we selected our catalyst of choice chitosan- $\mathrm{SO}_{3} \mathrm{H}$ $\left(\mathrm{CS}-\mathrm{SO}_{3} \mathrm{H}\right)$ and is prepared by following the reported procedure [10a]. To demonstrate the green protocol, we selected 2-mercaptoaniline (1) and simple benzaldehyde (2a) as the model reaction in presence of $\mathrm{CS}-\mathrm{SO}_{3} \mathrm{H}(50 \mathrm{~mol} \%)$ as a catalyst in ethanol at room temperature in presence of air balloon and achieved the cyclized compound $\mathbf{3}(\mathbf{a})$ in $60 \%$ yield (Table-1, entry-1). The analytical data of the obtained compound was in agreement with the reported data [11].

TABLE-1

OPTIMIZATION AND VARIATIONS (mol \%) OF THE

CATALYST AND SOLVENT EFFECT FOR THE SYNTHESIS OF 2-ARYL/HETEROARYL BENZOTHIAZOLES (3a)

\begin{tabular}{cccccc}
\hline Entry & Catalyst & $\begin{array}{c}\text { Mol } \\
(\%)\end{array}$ & Solvent & $\begin{array}{c}\text { Time } \\
(\mathrm{min})^{\mathrm{b}}\end{array}$ & $\begin{array}{c}\text { Yield } \\
(\%)^{\mathrm{a}}\end{array}$ \\
\hline 1 & $\mathrm{CS}-\mathrm{SO}_{3} \mathrm{H}$ & 50 & $\mathrm{EtOH}$ & 60 & 60 \\
2 & $\mathrm{CS}^{-S_{3} \mathrm{H}}$ & 30 & $\mathrm{CH}_{3} \mathrm{CN}$ & 50 & 65 \\
3 & $\mathrm{CS}_{3} \mathrm{SO}_{3} \mathrm{H}$ & 20 & PEG-400 & 45 & 70 \\
4 & $\mathrm{CS}^{-\mathrm{SO}_{3} \mathrm{H}}$ & 10 & THF & 40 & 75 \\
5 & $\mathrm{CS}^{-S_{3} \mathrm{H}}$ & 5 & Solvent less & 30 & 80 \\
6 & $\mathrm{CS}_{3}-\mathrm{SO}_{3} \mathrm{H}$ & 2 & Solvent less & 30 & 90 \\
7 & No catalyst & - & Solvent less & 600 & Trace \\
\hline
\end{tabular}

${ }^{\mathrm{a}}$ Isolated yields; ${ }^{\mathrm{b}} \mathrm{All}$ the reactions were performed at room temperature under oxygen balloon.

We further explored the synthetic protocol and extended the scope of the reaction by varying the catalyst loadings and studied the effect of various solvents towards the reaction profile. Among the attempted conditions $\mathrm{CS}-\mathrm{SO}_{3} \mathrm{H}(2 \mathrm{~mol} \%)$ under solvent free conditions at room temperature resulted the cyclized product in $90 \%$ yield with high selectivity (Table1 , entry-6). The increased mol $\%$ of the catalyst towards the reaction is not satisfactory as depicted in (Table-1, entry: 1-4). In the absence of catalyst no formation of the expected product was detected even after prolonged hours (Table-1, entry-7).

Encouraged by the above appended results, we further explored the synthetic protocol and the scope of the reaction by employing substituted aryl/heteroaryl aldehydes functionalized with electron-rich and electron-deficient groups. The results were depicted in (Table-2, entries 2 to 15).

Clearly, all reactions worked well irrespective of the substrates present on the aldehydes even with electron-with- 


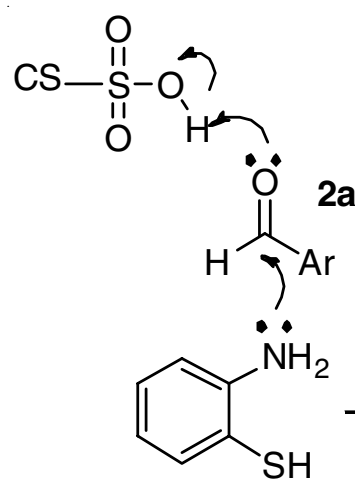

1<smiles>CCO</smiles><smiles>CSS(=O)(=O)[O-]</smiles>

$+$<smiles>BrC1CCN2CC1SSc1ccccc12</smiles><smiles>[Bi]C1Nc2ccccc2S1</smiles>

A
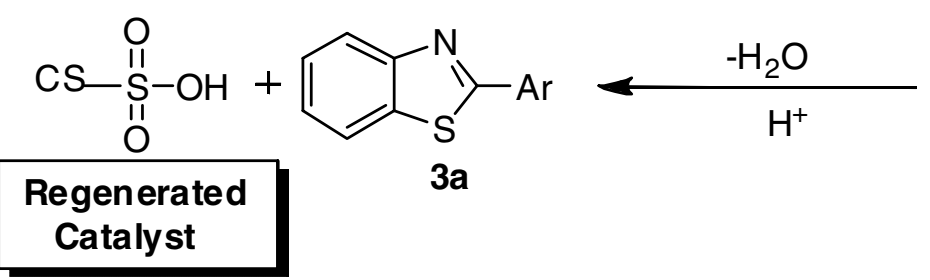<smiles>OC1([Al])Nc2ccccc2S1</smiles>

3a

Scheme-II: Sequential and plausible mechanistic pathway for the preparation of 2-aryl/heteroaryl benzothiazoles by using chitosan- $\mathrm{SO}_{3} \mathrm{H}$

\begin{tabular}{|c|c|c|c|c|}
\hline \multicolumn{5}{|c|}{$\begin{array}{c}\text { TABLE-2 } \\
\text { SYNTHESIS OF 2-ARYL/HETEROARYL BENZOTHIAZOLES } \\
\text { USING CS-SO }{ }_{3} \text { H AS A CATALYST UNDER SOLVENT } \\
\text { FREE CONDITIONS AT ROOM TEMPERATURE }\end{array}$} \\
\hline Entry & $\mathrm{R}-\mathrm{CHO}$ & Products $^{\mathrm{a}}$ & Time $(\min )^{\mathrm{b}}$ & 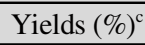 \\
\hline 1 & $\mathrm{C}_{6} \mathrm{H}_{5}$ & 3(a) & 30 & 90 \\
\hline 2 & 4- $\mathrm{CH}_{3} \mathrm{C}_{6} \mathrm{H}_{4}$ & 3(b) & 25 & 92 \\
\hline 3 & 4- $\mathrm{MeOC}_{6} \mathrm{H}_{4}$ & $3(c)$ & 25 & 89 \\
\hline 4 & $2-\mathrm{OHC}_{6} \mathrm{H}_{4}$ & 3(d) & 40 & 85 \\
\hline 5 & $4-\mathrm{BrC}_{6} \mathrm{H}_{4}$ & 3(e) & 30 & 90 \\
\hline 6 & $4-\mathrm{ClC}_{6} \mathrm{H}_{4}$ & $3(f)$ & 35 & 89 \\
\hline 7 & $4-\mathrm{FC}_{6} \mathrm{H}_{4}$ & $3(g)$ & 30 & 88 \\
\hline 8 & 4- $\mathrm{CF}_{3} \mathrm{C}_{6} \mathrm{H}_{4}$ & $3(h)$ & 35 & 89 \\
\hline 9 & $3-\mathrm{NO}_{2} \mathrm{C}_{6} \mathrm{H}_{4}$ & 3(i) & 40 & 87 \\
\hline 10 & 3-Br-4- $\mathrm{CH}_{3} \mathrm{C}_{6} \mathrm{H}_{3}$ & $3(\mathbf{j})$ & 30 & 92 \\
\hline 11 & 2-Naphthyl & $3(\mathbf{k})$ & 30 & 90 \\
\hline 12 & 2-Thiophenyl & 3(l) & 35 & 89 \\
\hline 13 & 3-Pyridyl & $3(\mathbf{m})$ & 30 & 92 \\
\hline 14 & 4-Pyridyl & 3(n) & 30 & 90 \\
\hline 15 & 2-Furanyl & $3(0)$ & 35 & 89 \\
\hline
\end{tabular}

${ }^{a}$ All Products were characterized by ${ }^{1} \mathrm{H}$ NMR, ${ }^{13} \mathrm{C}$ NMR and Mass spectra. ${ }^{b}$ The temperature for all the synthesized products is at room

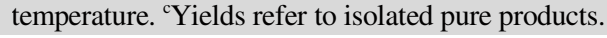

drawing groups. The process successfully achieved the 2-aryl/ heteroaryl benzothiazole derivatives (3a-o) (Table-2, entries 1-15) in good to excellent yields. After completion of the reaction the catalyst is easily separated by filteration and reused after activation at $120^{\circ} \mathrm{C}$ for $3 \mathrm{~h}$. The results obviously indicates no significant loss in its activity with that of fresh catalyst as depicted in Table-3.

A plausible mechanistic study is depicted in Scheme-II, showing the catalytic activation of the $\mathrm{CS}-\mathrm{SO}_{3} \mathrm{H}$. The catalyst

\begin{tabular}{|c|c|c|c|c|c|}
\hline \multicolumn{6}{|c|}{$\begin{array}{c}\text { TABLE-3 } \\
\text { REUSE OF THE CATALYST FOR THE SYNTHESIS OF } \\
\text { 2-ARYL/HETEROARYL BENZOTHIAZOLES }\end{array}$} \\
\hline Run & 1 & 2 & 3 & 4 & 5 \\
\hline Yield (\%) & 92 & 92 & 91 & 90 & 90 \\
\hline
\end{tabular}

can possibly act as a convenient proton source which initiates the nucleophilic attack of $\mathrm{NH}_{2}$ group on aldehyde carbonyl group activated by $\mathrm{CS}-\mathrm{SO}_{3} \mathrm{H}$ catalyst followed by the formation of an azomethine intermediate [A], followed by intramolecular cyclization to yield the intermediate $[\mathbf{B}]$, subsequent oxidation with $\mathrm{O}_{2}$ from air and expulsion of $\mathrm{H}_{2} \mathrm{O}$ to furnish the fully heteroaromatised product (3a) along with the regenerated CS$\mathrm{SO}_{3} \mathrm{H}$.

\section{Conclusion}

We have demonstrated that chitosan- $\mathrm{SO}_{3} \mathrm{H}$ catalyst is reusable and eco-friendly catalyst for the synthesis of 2-aryl/ heteroaryl benzothiazoles. Nevertheless, without doubt the new process has significantly improved compared to those in the known literature in terms of high selectivity, short reactions times, excellent yields, no thermal heating, no metal oxidant, large substrate scope and without any waste generation or byproduct formation. Further no column chromatography is required. The process designed focuses on being environment friendly under green chemistry aspects.

\section{ACKNOWLEDGEMENTS}

The authors thank the Management of Alekhya Drugs Pvt. Ltd., Vijayawada, India for encouragement and support. 


\section{REFERENCES}

1. E. Rodriguez, G.H.N. Towers and J.C. Mitchell, Phytochemistry, 15, 1573 (1976); https://doi.org/10.1016/S0031-9422(00)97430-2.

2. (a) T.D. Bradshaw and A.D. Westwell, Curr. Med. Chem., 11, 1009 (2004); https://doi.org/10.2174/0929867043455530.

(b) S.J. Hays, M.J. Rice, D.F. Ortwine, G. Johnson, R.D. Schwarz, D.K. Boyd, L.F. Copeland, M.G. Vartanian and P.A. Boxer, J. Pharm. Sci., 83, 1425 (1994);

https://doi.org/10.1002/jps.2600831013.

(c) C.J. Paget, K. Kisner, R.L. Stone and D.C. Delong, J. Med. Chem., 12, 1016 (1969); https://doi.org/10.1021/jm00306a011.

(d) D. Kumar, M.R. Jacob, M.B. Reynolds and S.M. Kerwin, Bioorg. Med. Chem., 10, 3997 (2002);

https://doi.org/10.1016/S0968-0896(02)00327-9.

(e) D. Alagille, R.M. Baldwin and G.D. Tamagnan, Tetrahedron Lett., 46, 1349 (2005);

https://doi.org/10.1016/j.tetlet.2004.12.111.

3. (a) B.A. Tuylu, H.S. Zeytinoglu and I. Isikdag, Biologia, 62, 626 (2007); https://doi.org/10.2478/s11756-007-0122-4.

(b) S.J. Choi, H.J. Park, S.K. Lee, S.W. Kim, G. Han and H.Y. Choo, Bioorg. Med. Chem., 14, 1229 (2006); https://doi.org/10.1016/j.bmc.2005.09.051.

(c) A.L. Loaiza-Pe'rez, V. Trapani, C. Hose, S.S. Singh, J. Trepel, M.F.G. Stevens, T.D. Bradshaw and E.A. Sausville, Mol. Pharmacol., 61, 13 (2002);

https://doi.org/10.1124/mol.61.1.13.

4. A.W. Hofmann, Ber. Dtsch. Chem. Ges., 12, 1126 (1879); https://doi.org/10.1002/cber.187901201293.

5. P. Jacobson and A. Frankenbacher, Ber. Dtsch. Chem. Ges., 24, 1400 (1891); https://doi.org/10.1002/cber.189102401247.

6. V.J. Majo, J. Prabhakaran, J.J. Mann and J.S. Dileep Kumar, Tetrahedron Lett., 44, 8535 (2003);

https://doi.org/10.1016/j.tetlet.2003.09.138.

7. (a) R. Breslow, Acc. Chem. Res., 13, 170 (1980); https://doi.org/10.1021/ar50150a002.

(b) J.H. Clark and D.J. Macquarrie, Green Chemistry and Technology, Blackwell, Abingdon (2002).

(c) P. Gupta and S. Paul, Catal. Today, 236, 153 (2014);

https://doi.org/10.1016/j.cattod.2014.04.010.

(d) M. Lee, B.-Y. Chen and W. Den, J. Appl. Sci. (Faisalabad), 5, 1272 (2015);

https://doi.org/10.3390/app5041272.

8. (a) A. Loupy, F. Maurel and A. Sabatié-Gogová, Tetrahedron, 60, 1683 (2004);

https://doi.org/10.1016/j.tet.2003.11.042.

(b) B.K. Pchelka, A. Loupy and A. Petit, Tetrahedron, 62, 10968 (2006); https://doi.org/10.1016/j.tet.2006.08.067.

(c) M.A.P. Martins, C.P. Frizzo, D.N. Moreira, L. Buriol and P. Machado, Chem. Rev., 109, 4140 (2009);

https://doi.org/10.1021/cr9001098. (d) G.W.W. Cave, C.L. Raston and J.L. Scott, Chem. Commun., 21, 2159 (2001);

https://doi.org/10.1039/b106677n.

(e) K. Tanaka and F. Toda, Chem. Rev., 100, 1025 (2000);

https://doi.org/10.1021/cr940089p.

9. (a) M.G. Peter, eds.: S. De Baets, E. Vandamme and A. Steinbüchel, Chitin and Chitosan in Fungi, In: Polysaccharides II: Polysaccharides from Eukaryotes, Wiley-VCH, Weinheim, vol. 6, p. 123-132 (2002).

(b) V.E. Tikhonov, E.A. Stepnova, V.G. Babak, I.A. Yamskov, J. PalmaGuerrero, H.-B. Jansson, L.V. Lopez-Llorca, J. Salinas, D.V. Gerasimenko, I.D. Avdienko and V.P. Varlamov, Carbohydr. Polym., 64, 66 (2006); https://doi.org/10.1016/j.carbpol.2005.10.021.

10. (a) K. Khan and Z.N. Siddiqui, Ind. Eng. Chem. Res., 54, 6611 (2015); https://doi.org/10.1021/acs.iecr.5b00511.

(b) B.V.S. Reddy, A. Venkateswarlu, G.N. Reddy and Y.V.R. Reddy, Tetrahedron Lett., 54, 5767 (2013);

https://doi.org/10.1016/j.tetlet.2013.07.165.

(c) J. Safari, Z. Zarnegar, M. Sadeghi and F. Azizi, Curr. Org. Chem., 20, 2926 (2016); https://doi.org/10.2174/1385272820666160805112208.

11. G. Evindar and R.A. Batey, J. Org. Chem., 71, 1802 (2006); https://doi.org/10.1021/jo051927q.

12. H. Deng, Z. Li, F. Ke and X. Zhou, Chem. Eur. J., 18, 4840 (2012); https://doi.org/10.1002/chem.201103525.

13. A.-M. Osman and I. Bassiouni, J. Org. Chem., 27, 558 (1962); https://doi.org/10.1021/jo01049a051.

14. H. Sharghi and O. Asemani, Synth. Commun., 39, 860 (2009); https://doi.org/10.1080/00397910802431214.

15. Z. Duan, S. Ranjit and X. Liu, Org. Lett., 12, 2430 (2010); https://doi.org/10.1021/o1100816g.

16. T. Itoh and T. Mase, Org. Lett., 9, 3687 (2007); https://doi.org/10.1021/o17015737.

17. J.K. Huang, J. Chan, Y. Chen, C.J. Borths, K.D. Baucom, R.D. Larsen and M.M. Faul, J. Am. Chem. Soc., 132, 3674 (2010); https://doi.org/10.1021/ja100354j.

18. C. Praveen, K.H. Kumar, D. Muralidharan and P.T. Perumal, Tetrahedron, 64, 2369 (2008): https://doi.org/10.1016/j.tet.2008.01.004

19. Y. Sun, H. Jiang, W. Wu, W. Zeng and X. Wu, Org. Lett., 15, 1598 (2013); https://doi.org/10.1021/ol400379z.

20. T. Yamamoto, K. Muto, M. Komiyama, J. Canivet, J. Yamaguchi and K. Itami, Chem. Eur. J., 17, 10113 (2011); https://doi.org/10.1002/chem.201101091.

21. M. Kodomari, Y. Tamaru and T. Aoyama, Synth. Commun., 34, 3029 (2004); https://doi.org/10.1081/SCC-200026663.

22. T.G. Deligeorgiev, Dyes Pigments, 12, 243 (1990); https://doi.org/10.1016/0143-7208(90)85016-H.

23. P.B. Gorepatil, Y.D. Mane and V.S. Ingle, Synlett, 24, 2241 (2013); https://doi.org/10.1055/s-0033-1339758.

24. (a) B.S. Bose, K. Mukkanti and H. Venkatasubramanian, Lett. Org. Chem., 14, 353 (2017); https://doi.org/10.2174/1570178614666170329151935.

(b) B.S. Bose, K. Mukkanti and H. Venkatasubramanian, Der Pharma Chem., 6, 326 (2014). 